\title{
"La médecine moléculaire prend chaque jour plus d'importance»
}

\section{Interview: Matthias Scholer ${ }^{c}$}

${ }^{a}$ Dr en Sciences de la Vie, Cheffe de projet en Bioinformatique Clinique, SIB Institut Suisse de Bioinformatique;

${ }^{b}$ Dr en Biotechnologie, Senior Director Contract R\&D Unit, Hôpital universitaire de Bâle; ${ }^{c}$ Rédacteur "Bulletin des médecins suisses» en ligne et papier

De nos jours, les conseils interdisciplinaires en oncologie moléculaire font partie de la routine quotidienne des grandes institutions. La médecine personnalisée s'apprête toutefois à poser de nouveaux défis pour les médecins de famille au niveau des soins de base. Ils ont eux aussi besoin de connaissances étendues sur les modifications pathologiques survenant au niveau des gènes et sur les possibilités de thérapies moléculaires existantes.

\begin{abstract}
C'est notamment en oncologie que le profilage moléculaire d'un échantillon de tissu acquiert de plus en plus d'importance. Pour des médecins qui ne sont pas confrontés à de telles technologies dans leurs activités quotidiennes, ce sujet est souvent une boîte noire dont ils se sentent exclus. A juste titre?

Luca Quagliata (LQ): Non, certainement pas. Tous peuvent et devraient comprendre en théorie le processus du séquençage du génome. Cette connaissance constitue en effet la base qui permet de tirer, dans la pratique de tous les jours, des conclusions correctes à partir des résultats d'un rapport pathologique et de s'orienter vers un traitement optimal.
\end{abstract}

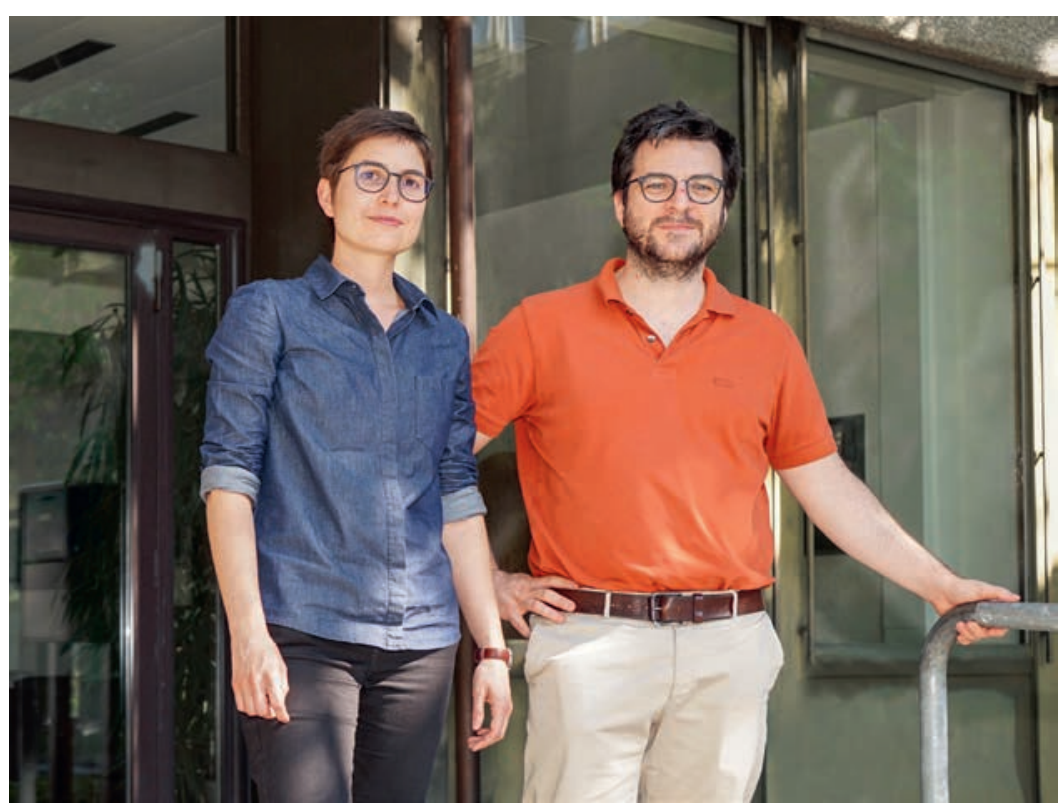

Aitana Lebrand et Luca Quagliata s'engagent pour la transmission des connaissances dans le domaine de la médecine moléculaire.
Aitana Lebrand ( $A L)$ : C'est la raison pour laquelle l'un des objectifs que nous poursuivons dans le cadre d'un groupe de travail suisse est la standardisation des rapports de pathologie moléculaire. Nous ne souhaitons pas de comptes rendus de vingt pages mais des rapports faciles à lire et globalement compréhensibles. Une base de connaissances en médecine moléculaire est toutefois nécessaire pour pouvoir en exploiter de façon optimale les informations.

\section{Quel rôle joueront les médecins de famille}

pour les patients qui font l'objet d'un traitement ciblé contre le cancer?

$L Q$ : D'une part, l'importance de leur rôle en tant que gatekeepers va augmenter. Celui-ci commence avec la récolte d'informations détaillées lors de l'anamnèse. En effet, la plupart des modifications génétiques sont causées par des facteurs familiaux ou externes tels que l'environnement professionnel ou le style de vie, qui inclut par exemple le tabagisme. Par conséquent, les informations sur un patient revêtent une importance cruciale pour la pose d'un diagnostic et le choix d'un traitement optimal. D'autre part, le suivi des patients après un traitement personnalisé contre le cancer accroît la tâche des médecins de famille. Comme le taux de survie obtenu grâce à ces nouveaux traitements augmente dans de nombreux cas, le lien entre le cabinet du médecin et le patient aura tendance à s'intensifier aussi à l'avenir. C'est pourquoi il est important que les médecins de famille sachent quels médicaments se trouvent sur le marché dans le domaine du traitement anticancéreux personnalisé, comment ils fonctionnent et à quoi il faut prêter attention tout au long du suivi du patient. 


\section{Comment les médecins peuvent-ils acquérir ces connaissances?}

$A L$ : Notre groupe de travail est une plateforme idéale pour comprendre les besoins cliniques dans le domaine de l'oncologie personnalisée tout en offrant des possibilités de formation continue adaptées. Sur la base de ces discussions, le SIB lance cet automne un CAS en oncologie moléculaire personnalisée, en collaboration avec les Hôpitaux universitaires de Bâle et de Lausanne.

\section{Un cours exclusivement destiné aux oncologues, donc?}

$A L$ : Non. Le cours s'adresse à tous les professionnels impliqués dans la pose d'un diagnostic et la prise d'une décision thérapeutique - des médecins généralistes aux oncologues, en passant par les pathologistes, les bioinformaticiens ou encore les généticiens. L'objectif de ce cours est de présenter dans le détail les technologies, les processus, les possibilités mais aussi les limitations de la pathologie moléculaire et des traitements personnalisés. Chaque personne impliquée saura ainsi ce qui est important pour chaque étape du processus et comment elle peut contribuer, par son propre travail, au meilleur résultat possible.

\section{Jetons à présent un œil sur les institutions hospitalières et la recherche. Quelles sont les activités prévues dans ce domaine?}

$A L$ : Le but à moyen terme est d'harmoniser et de standardiser les procédés de biologie moléculaire ainsi que l'interprétation des résultats de laboratoire. En effet, le caractère pathogène de nombreuses variantes de gènes étant encore incertain, chaque hôpital est amené à interpréter ces dernières de façon différente. Or, il est dans l'intérêt de tous qu'une mutation soit interprétée de manière standardisée dans toute la Suisse. A cet effet, les cliniques universitaires et les hôpitaux compétents en matière de recherche se sont rassemblés, sous l'égide du SIB et de notre groupe de travail suisse, afin de mettre sur pied, au cours des trois prochaines années, une base de données qui garantisse que tous les partenaires impliqués aient accès à des informations de haute qualité et avec lesquelles ils peuvent travailler. Ce projet a été approuvé dans le cadre de l'initiative Swiss Personalized Health Network.
LQ: Sur la base de ces données, un comité d'experts peut aboutir à un consensus sur la pathogénicité de chaque variante de gène. Et comme ce sont aujourd'hui des équipes pluridisciplinaires qui travaillent de concert à la pose d'un diagnostic et à la recherche d'un traitement, il est crucial que toutes les personnes impliquées aient des connaissances de base en médecine moléculaire. Ce n'est qu'ainsi que les équipes peuvent adopter un langage commun.

\section{Une tendance transposable à différents domaines de la médecine?}

$A L$ : Absolument. Il nous suffit de considérer la génétique médicale chez le nouveau-né, ou bien le domaine de l'infectiologie. On peut en outre présager qu'à l'avenir, la demande en matière de tests génétiques pour des facteurs de risque va augmenter auprès des médecins de famille. Toutes ces possibilités d'applications requièrent une compréhension de base de méthodes telles que le séquençage du génome. En effet, la médecine moléculaire prend chaque jour plus d'importance.

\section{Le CAS en oncologie moléculaire personnalisée}

Premier du genre en Suisse, le CAS (certificat d'études avancées) en oncologie moléculaire personnalisée est ouvert à tout professionnel de la santé qui souhaiterait acquérir des connaissances sur les méthodes de génération, d'analyse et d'interprétation du profil moléculaire de patients ou simplement étendre ses compétences et se confronter aux défis en la matière. La première session débute en novembre 2018. Inscription et informations détaillées: www.pmo.unibas.ch

Crédit photo

Matthias Scholer 